Classification

Physics Abstracts

$71.55 \mathrm{~J}-71.25-71.50-05.60$

\title{
Dimensional and magnetic field effects on localization
}

\author{
H. Kunz and B. Souillard (*) \\ Institut de Physique Théorique, EPF-Lausanne, CH-1006 Lausanne, Switzerland \\ $\left(^{*}\right)$ Centre de Physique Théorique, Ecole Polytechnique, F 91128 Palaiseau, France \\ (Reçu le 20 octobre 1981, accepté le 30 novembre 1981)
}

\begin{abstract}
Résumé. - Nous discutons la localisation d'une particule dans un potentiel aléatoire en termes d'une probabilité de retour et d'un temps de séjour. Au second ordre dans le potentiel, nous trouvons des comportements qualitativement différents en dimension un, deux ou trois. En particulier en dimension deux, nous trouvons des états ni localisés, ni vraiment étendus. Ces résultats apparaissent aussi pour des systèmes avec désordre non diagonal. En présence d'un champ magnétique, nos résultats suggèrent une complète localisation à faible concentration d'impuretés ou à grand champ.
\end{abstract}

\begin{abstract}
We discuss the localization of a particle in a random potential in terms of a probability of return and a sojourn time. At the second order in the potential, we find qualitatively different behaviours in dimension one, two and three. In particular for dimension two, we find states neither localized, nor truly extended. These results hold also for systems with off-diagonal disorder. In the presence of a magnetic field, our results suggest complete localization at weak concentration of impurities or at large field.
\end{abstract}

Since Abrahams et al. [1] proposed on the basis of their scaling theory that all states should be localized in a two-dimensional system and that the static conductivity should also vanish, a number of authors have reexamined the situation by various methods. Whereas much of the subsequent work [2] confirmed their predictions, various numerical and theoretical studies persistently suggested the existence of a transition. First, there were the renormalization group computation of Lee [3] and the recursion-decimation results of Stein and Krey [4] and F. Yonezawa et al. [5]. Then, we developed a perturbative approach allowing to study the nature of the states for weak disorder and showing in the case of dimension two, that states were neither localized, nor fully extended. These results were presented repeatedly in various meetings [6] since 1980. We give here some details about the method and present new results. Recently, Pichard and Sarma [7] obtained also evidence in dimension two for quasi-extended states, using a phenomenological finite size scaling approach. Meanwhile Kaveh and Mott [8], on the basis of a very suggestive treatment, obtained also evidence for quasi-extended states, but showed that this was compatible with a vanishing conductivity, as predicted by Abrahams et al.

To make a precise enough distinction between various kinds of extended or localized states, we will introduce two useful quantities : the time of sojourn and the probability of return of a 
quantum particle. More precisely, let $\Lambda$ designate a finite region of space (a ball around the origin for example). If the particle is initially in the normalized state $\varphi$, and has an energy in the range $A=[\alpha, \beta]$, then the time spent by the particle in the region $\Lambda$ will be given by

$$
T_{\Lambda}=\lim _{\varepsilon \rightarrow 0^{+}} T_{\Lambda}(\varepsilon)=\lim _{\varepsilon \rightarrow 0^{+}} \int_{0}^{\infty} \mathrm{d} t \mathrm{e}^{-\varepsilon t}\left\|P_{\Lambda} \mathrm{e}^{-i t H} E(A) \varphi\right\|^{2}
$$

where $P_{A}$ is the projector on the region $\Lambda$, and $E(A)$ the spectral projector of $H$ on the interval of energy $A$.

If we choose

$$
\varphi(x)=\left\{\begin{array}{ll}
1 & x \in \Lambda \\
0 & x \notin \Lambda
\end{array},\right.
$$

then the particle starts in $\Lambda$, and $T_{\Lambda}$ measures the time of sojourn of the particle in $\Lambda$. We are also interested by the probability of return of the particle in $\Lambda$, namely :

$$
R_{\Lambda}=\lim _{T \rightarrow \infty} \frac{1}{T} \int_{0}^{T} \mathrm{~d} t\left\|P_{\Lambda} \mathrm{e}^{-i t H} E(A) \varphi\right\|^{2}=\lim _{\varepsilon \rightarrow 0^{+}} \varepsilon T_{\Lambda}(\varepsilon) .
$$

Three situations are then possible :

A) $R_{\Lambda} \neq 0$ and $T_{\Lambda}=\infty$

B) $R_{A}=0$ but $T_{A}=\infty$

C) $R_{A}=0$ and $T_{A}<\infty$.

In the first case (A), the particle does not diffuse out of the finite region $\Lambda$ and therefore remains localized. In this case, we could conclude that states in the energy range $A$ are localized. Strictly speaking, we cannot exclude the possibility of a superposition of localized and extended states by such a criterion, but we will assume, as usually done, that such a situation does not occur. Note that this criterion was the one used by Anderson in his original paper on localization.

The third case $(C)$ indicates diffusion of the wave packet, and we will say that if we are in such a case, we have extended states in the energy range $A$. Both situations, $(\mathrm{A})$ and $(\mathrm{C})$, are familiar in quantum mechanics and can be shown to correspond (under some hypothesis) to the presence of what is called pure point spectrum for the Hamiltonian in case (A) and to an absolutely continuous spectrum is case $(\mathrm{C})$.

The middle case (B) has not occurred up to now in quantum mechanics. Under some hypothesis (rather strong admittedly), it can be shown to correspond to the so-called singular continuous spectrum. Physically a possible picture of the situation is the following : the particle leaves the region $\Lambda$ for some time, then comes back in it, then leaves it again, comes back and so forth, until finally it really diffuses away out of this region. Its successive returns in the region $\Lambda$ will be so frequent that the time spent by it in the region will be infinite. A regular but very slow diffusion out of the region might also be possible. The eigenfunctions in this energy range are not square integrable. They may decay to zero at large distances like $r^{-\eta}$ for example. They might also have no unique asymptotic behaviour, like in the case of a particle moving in a quasi-periodic potential, as found by Avron and Simon [10]. It is clear that, in this case, states are extended to some extent, but localized from another point of view. They can be called quasi-extended.

We would like to show now that all these situations appear for a quantum particle in a weak random potential, when its energy is in the unperturbed band and when we vary the dimension of the space. Case (A) appears in one dimension, or in three dimensions, when we add a constant magnetic field to the system. We conclude that in this case all states should be localized. 
Case (B) appears in dimension two and suggests the existence of a mobility edge separating localized states from quasi-extended states.

Case $(C)$ is the usual one in dimension three and shows that usual extended states should be present in three dimensions at least far from the band edges. The situation for a particle moving on the Bethe lattice is the same, in agreement with a rigorous analysis [9]. This can be expected, since the Bethe lattice corresponds in some sense to the situation in an infinite number of dimensions.

What we will do is to compute to lowest order in the potential the time of sojourn and the probability of return, in these various situations. In this way, we can certainly determine the stability of the usual extended states, with respect to the random potential at least when the energy stays in the spectrum of the unperturbed Hamiltonian. But the result itself suggests the nature of the spectrum of the Hamiltonian itself. In order to do so, we express $T_{A}$ and $R_{A}$ in terms of the resolvent of the Hamiltonian

$$
T_{\Lambda}(\varepsilon)=\frac{1}{2 \pi} \int_{-\infty}^{+\infty} \mathrm{d} e\left\|P_{\Lambda} R\left(e+i \frac{\varepsilon}{2}\right) E(A) \varphi\right\|^{2}
$$

and it can be shown that

$$
R_{\Lambda}(\varepsilon)=\lim _{\varepsilon \rightarrow 0^{+}} \frac{\varepsilon}{2 \pi} \int_{A} \mathrm{~d} e\left\|P_{A} R\left(e+i \frac{\varepsilon}{2}\right) \varphi\right\|^{2}
$$

where

$$
R(e+i \varepsilon)=(e+i \varepsilon-H)^{-1}
$$

and where the projector on an interval $A$ of energies, $E(A)$ is given by

$$
E(A)=\lim _{\varepsilon \rightarrow 0^{+}}-\pi^{-1} \operatorname{Im} \int_{A} \mathrm{~d} e R(e+i \varepsilon) .
$$

For simplicity, let us look to the lattice case

$$
H=-\Delta+V
$$

where $V=\{V(x)\}$ is a collection of independent random variables with a common distribution.

Then, putting the particle initially at the origin (i.e. $\varphi(x)=\delta(x))$ we find for the average time spent by the particle at the origin (i.e. $\Lambda=\{0\}$ in the formulas)

$$
\left\langle T_{\Lambda}(\varepsilon)\right\rangle=\frac{1}{2 \pi} \int_{-\infty}^{+\infty} \mathrm{d} e\left\langle\left|\left(R\left(e+i \frac{\varepsilon}{2}\right) \psi_{A}\right)(0)\right|^{2}\right\rangle
$$

with

$$
\psi_{A}(x)=E_{x_{0}}(A)=\left(\delta_{x}, E(A) \delta_{0}\right)
$$

Since (as can be checked order by order in perturbation)

$$
\lim _{\varepsilon \rightarrow 0^{+}}\left|\left\langle\left(R\left(e+i \frac{\varepsilon}{2}\right) \psi_{A}\right)(0)\right\rangle\right|<\infty
$$


we have to lowest order in the potential

$$
\lim _{\varepsilon \rightarrow 0^{+}}\left\langle T_{\Lambda}(\varepsilon)\right\rangle=\text { finite terms }+\lim _{\varepsilon \rightarrow 0^{+}} \int_{-\infty}^{+\infty} \frac{\mathrm{d} e}{2 \pi}\left\langle\left|\left(R^{(1)}\left(e+i \frac{\varepsilon}{2}\right) \psi_{A}^{(0)}\right)(0)\right|^{2}\right\rangle
$$

where $R^{(1)}$ is the first order contribution to $R$

$$
R^{(1)}=R^{(0)} V R^{(0)}
$$

and $\psi_{A}^{(0)}(x)=E_{x_{0}}^{(0)}(A)$ the lowest order contribution to $\psi_{A}(x)$

$$
\begin{aligned}
R_{e+i \varepsilon}^{(0)}(x, y) & =\left(\frac{1}{2 \pi}\right)^{d} \int_{-\pi}^{\pi} \frac{\mathrm{d} k_{1} \ldots \mathrm{d} k_{d} \exp i \mathbf{k} \cdot(\mathbf{x}-\mathbf{y})}{e+i \varepsilon-\varepsilon(k)} \\
\psi_{A}^{(0)}(x) & =\left(\frac{1}{2 \pi}\right)^{d} \int_{-\pi}^{\pi} \mathrm{d} k_{1} \ldots \mathrm{d} k_{d} \exp i \mathbf{k} \cdot \mathbf{x} \\
\varepsilon(k) \leqslant \beta & =2 \sum_{1}^{d}\left(1-\cos k_{j}\right) .
\end{aligned}
$$

We then find that the possibly singular contribution to (I) is given by

$$
\left\langle V^{2}(x)\right\rangle \int_{-\infty}^{+\infty} \mathrm{d} e \sum_{y}\left|R_{e+i \frac{\varepsilon}{2}}^{(0)}(y, 0)\right|^{2}\left|C_{e+i \frac{\varepsilon}{2}}(y)\right|^{2}
$$

where

$$
C_{e+i \frac{\varepsilon}{2}}(y)=\left(\frac{1}{2 \pi}\right)^{d} \int_{\substack{-\pi \\ \alpha}}^{\pi} \mathrm{d} k_{1} \ldots \mathrm{d} k_{d} \frac{\exp i \mathbf{k} \cdot \mathbf{y}}{e+i \frac{\varepsilon}{2}-\varepsilon(k)} .
$$

When $y$ is fixed, both $R_{e+i \varepsilon}^{(0)}(y, 0)$ and $C_{e+i \frac{\varepsilon}{2}}(y)$ have finite limits when $\varepsilon \rightarrow 0^{+}$. Therefore, the only singular behaviour comes from the long distance behaviour of $R_{e+i \frac{\varepsilon}{2}}^{(0)}(y)$ and $C_{e+i \frac{\varepsilon}{2}}(y)$.

But we know that for large $|y|$

when

$$
\left|C_{e+i \frac{\varepsilon}{2}}^{0}(y)\right| \sim\left|R_{e+i \frac{\varepsilon}{2}}^{0}(y)\right| \sim \frac{\mathrm{e}^{-\frac{\varepsilon}{2}|y|}}{|y|^{\frac{d-1}{2}}}
$$

$$
0<\alpha<e<\beta<4 d,
$$

whereas both quantities tend exponentially to zero when $e$ is outside the unperturbed band.

Therefore, the singular part is given by

$$
\int_{\alpha}^{\beta} \mathrm{d} e \int_{1}^{\infty} \mathrm{d} x x^{d-1} \frac{\exp (-2 \varepsilon x)}{x^{2(d-1)}} \simeq\left\{\begin{array}{lll}
\frac{A_{1}}{\varepsilon} & \text { if } & d=1 \\
A_{2} \ln \varepsilon & \text { if } & d=2 \\
A_{3} & \text { if } & d \geqslant 3
\end{array}\right.
$$


when

$$
0<\alpha \leqslant \beta<4 d
$$

And we conclude that

A) $R_{\{0\}}=\lim _{\varepsilon \rightarrow 0^{+}} \varepsilon T_{\{0\}}(\varepsilon) \neq 0$ in 1 dimension, and $T_{\{0\}}=\infty$

B) $R_{\{0\}}=\lim _{\varepsilon \rightarrow 0^{+}} \varepsilon \ln \varepsilon=0$ in 2 dimensions

but $\quad T_{\{0\}}=\infty$

C) $R_{\{0\}}=0$ and $T_{\{0\}}<\infty$ in 3 dimensions, or more.

A more careful analysis shows that these conclusions remain valid under more general circumstances : correlated potentials, purely off-diagonal disorder, and constrained diagonal and off-diagonal disorder of the phononic type. We can also see that we could produce usual extended states even in one dimension by taking for the unperturbed Hamiltonian, a matrix with slow power fall-off of the off-diagonal elements.

In the Bethe lattice case, if $K+1$ is the coordination number we see that

$$
\left|C_{e+i \varepsilon}(y)\right| \sim\left|R_{e+i \varepsilon}^{0}(y)\right| \sim K^{-\frac{|y|}{2}} \mathrm{e}^{-\frac{\varepsilon}{2}|y|} .
$$

For large $|y|$, and since there are $K^{|y|}$ sites distant from the origin on this lattice we see that the singular term in this case goes like

$$
\lim _{\varepsilon \rightarrow 0^{+}} \sum_{|y|=1}^{\infty} K^{-|y|} \exp (-2 \varepsilon|y|)<\infty
$$

as soon as $K>1(K=1$ is the one-dimensional case).

So that $R_{\{0\}}=0$, but $T_{\{0\}}<\infty$, in this case, in agreement with a rigorous analysis [9]. All these conclusions remain valid in the continuous case, under rather weak conditions on the correlations of the potential if we restrict the energy to strictly positive values. In this case it is also quite interesting to discuss the situation for a particle in an external constant magnetic field, in three dimensions.

In cylindrical coordinates $(z, r, \grave{\varphi})$, the unperturbed propagator is given by

$$
R^{(0)}\left(z^{\prime} r^{\prime} \varphi^{\prime} \mid z r \varphi\right)=\frac{i}{2} \sum_{n=0}^{\infty} \sum_{m=-\infty}^{+\infty} \overline{\psi_{m n}\left(r^{\prime}, \varphi^{\prime}\right)} \psi_{m n}(r, \varphi) \frac{\exp i \varepsilon_{m n}\left|z-z^{\prime}\right|}{\varepsilon_{m n}}
$$

where

$$
\begin{aligned}
\varepsilon_{m n} & =\left[\left(e-e_{m n}\right)+i \varepsilon\right]^{1 / 2} \\
e_{m n} & =\omega\left[n+m \theta(m)+\frac{1}{2}\right] \\
\omega & =\text { Larmor frequency } \\
\theta(m) & = \begin{cases}1 & m \geqslant 0 \\
0 & m<0\end{cases}
\end{aligned}
$$

$\psi_{m n}(r, \varphi)$ being a set of special functions forming a basis of $L_{2}(r \mathrm{~d} r, \mathrm{~d} \varphi)$.

This propagator is an infinite (but convergent) sum of propagators of one-dimensional type in the $z$ direction

$$
\frac{i}{2} \varepsilon_{m n}^{-1} \exp \left(i \varepsilon_{m n}\left|z-z^{\prime}\right|\right)
$$


It is perhaps not too surprising therefore that one finds after some lengthy calculation that to lowest order in the potential the situation is the same as in the one-dimensional case without a magnetic field :

$$
T_{\Lambda}=\infty \text { and } R_{\Lambda} \neq 0
$$

if the energy range $A$ is inside any of the unperturbed bands $\left(\left[\varepsilon_{m, n}, \varepsilon_{m, n+1}\right]\right.$ for example).

We conclude that in this case, all states should be localized, for small concentration of impurities or large field. Although high order contributions might change this result, it can be understood intuitively by noting that the magnetic field localizes free particles in a Gaussian way in the plane perpendicular to the direction of the field, so that the particle can only diffuse in the direction parallel to the field. Fluctuations of the random potential in this direction, like in the usual one-dimensional problem, forbid its diffusion in this direction, so that ultimately it is completely localized.

This conclusion might be doubted, since many authors have computed a non zero conductivity for such a system, starting from a Boltzmann type approach. Their result might be valid because practically the localization length (that we have not estimated) is huge compared to the Boltzmann mean free path. Therefore, the localization effect might be undetectable, except at very large fields. A more detailed investigation, however, is needed to elucidate these localization effects on the conductivity.

\section{References}

[1] Abrahams, E., Anderson, P. W., Licciardello, D. C. and Ramakrishnan, T. V., Phys. Rev. Lett. 42 (1979), 673.

[2] Wegner, F., Z. Phys. B 35 (1979), 207.

Goetze, W., Prelovsek, P. and Wölfle, P., Solid State Commun. 30 (1979) 369.

Gorkov, L. P., Larkin, A. I. and KhmelnitZKiI, D. E., Jett. P. Lett. 30 (1979), 229.

[3] LeE, P. A., Phys. Rev. Lett. 42 (1979) 1492.

[4] Stein, J. and Krey, U., Z. Phys. B 34 (1979), 287.

[5] Tsujino, K., Tokunaga, A., Yamamoto, M., Yonezawa, F. J. Non Cryst. Solids 35 (1980), 29.

[6] Kunz, H. and Soulllard, B., Helv. Phys. Acta 53 (1980), 327.

1980 Gwatt Workshop on disordered systems. Proceedings.

1981 International Conferences on Disordered Systems (Rome). Proceedings.

[7] Pichard, J. L. and Sarma, G., J. Phys. C. 14 (1981) L 127 and prepint submitted to J. Phys. C.

[8] Kaveh, M. and Mott, N. F., J. Phys. C. 14 (1981), L 413

[9] Kunz, H. and Souillard, B., The mobility edge on the Bethe lattice, Preprint.

[10] AVron, M. and Simon, B., private communication. 